\title{
Polarimetry of R Aqr and PN M2-9
}

\author{
Silvana G. Navarro ${ }^{1}$, Laurence Sabin ${ }^{1}$, Julio Ramírez ${ }^{2}$ \\ and David Hiriart ${ }^{2}$ \\ ${ }^{1}$ Instituto de Astronomía y Meteorología, Universidad de Guadalajara \\ email: silvana@astro.iam.udg.mx \\ ${ }^{2}$ Instituto de Astronomía, Universidad Nacional Autónoma de México, \\ email: julio@astroscu.unam.mx
}

\begin{abstract}
The bipolar or more complex morphology observed in planetary nebulae have been explained by two principal hypothesis: by the existence of a companion and an accreting disk or by the effects of magnetic field, (or a combination of both). Symbiotics are binary systems and some of them show morphologies similar to those observed on planetary nebulae. This fact could support the binary hypothesis for PNe. We have therefore performed polarimetric observations of symbiotic systems and some planetary nebulae in order, first to detect linear polarisation with POLIMA at the San Pedro Mártir observatory, and ultimately to prove the existence and physical properties of those disks. We present here the first results of a project dedicated to the analysis of the polarisation observed in evolved objects starting with the PN M2-9 and R Aqr.
\end{abstract}

Keywords. Polarimetry, planetary nebulae, symbiotic systems

\section{Introduction}

Polarization due to dust scattering is a useful tool to determine the geometry of extended envelopes of Symbiotic systems and proto-planetary nebulae (Scarrott \& Scarrott, 1995; Gledhill, 2005). It is also used to trace the circumstellar disk and jets/outflows present in such objects. M2-9 is a widely studied bipolar PN, it shows two coaxial shells and a series of dusty blobs, located simetrically on both lobes. Castro-Carrizo et al. 2012 found two ring-shaped structures at the center of this object giving evidence of their binary nature. R Aqr is a symbiotic system formed by a Mira variable (287 days period) and a white dwarf. It has two bipolar and co-axial shells with kinematical ages: 185 and 650 years (Solf \& Ulrich, 1985). A high collimated structure (jet) is observed in this object, with bright knots along the jet that evolve in position, brightness and size. UV observations reveal the nature of this jet-like structure (Nichols \& Slavin, 2009) They show that the shape of the emission lines is consistent with an ejecta model of a bow shock, supporting the existence of an accretion disk as the origin of the ejected material.

\section{Observations and Results}

We observed these objects with the polarimeter POLIMA on the $84 \mathrm{~cm}$ telescope of San Pedro Mrtir B. C. We used two narrow and two wide filters: $\mathrm{H} \alpha\left(\lambda_{c}=6564 \AA, \delta \lambda=72 \AA\right.$, H6819 (6819 А, $86 \AA), \operatorname{Gr}(6550 \AA, 900 \AA)$ and Gg (4930 A, $700 \AA)$. The latest are ThuanGunn filters. In the case of planetary nebulae M2-9, we determine the polarisation over both lobes, at the regions showed in figure 1a. In this object we detected a considerable polarisation, specially in the regions near to the central object. The polarisation angle does not change notably always near 80 degrees except at the extreme positions E1 and E2 (see table 1). In table 2 we present the polarisation measurements at the center and at the knots observed in $\mathrm{R}$ Aqr. The measured polarisation is low and its value depends 
Table 1. Polarisation measured in M2-9 over the regions showed in figure 1a

\begin{tabular}{ccccccc}
\hline Position & $\mathbf{P}(\%) \mathbf{H} \alpha$ & Angle & $\mathbf{P}(\%) \mathbf{G r}$ & Angle & $\mathbf{P}(\%) \mathbf{G g}$ & Angle \\
\hline Center & $4.6 \pm 0.3$ & $80 \pm 2$ & $4.3 \pm 0.2$ & $63 \pm 1.5$ & $3.9 \pm 0.3$ & $67 \pm 2$ \\
1N & $8.0 \pm 0.5$ & $84 \pm 3$ & $6.6 \pm 0.5$ & $73 \pm 2$ & $6.6 \pm 0.9$ & $69.6 \pm 3$ \\
2N & $20.6 \pm 0.7$ & $72 \pm 2$ & $18.9 \pm 0.7$ & $72.4 \pm 1.4$ & $19.5 \pm 1.5$ & $82.2 \pm 1.8$ \\
3S & $11.1 \pm 0.5$ & $80 \pm 2$ & $16.4 \pm 0.6$ & $76.4 \pm 1.4$ & $17.2 \pm 1.2$ & $105.4 \pm 1.7$ \\
$4 \mathrm{~S}$ & $10.2 \pm 0.5$ & $79 \pm 2$ & $9.2 \pm 0.6$ & $70.3 \pm 1.7$ & $8.1 \pm 1$ & $75 \pm 3$ \\
E1 & $16 \pm 2$ & $90 \pm 3$ & $13.3 \pm 0.4$ & $75.1 \pm 1.3$ & $21.6 \pm 4$ & $176 \pm 4$ \\
E2 & $4 \pm 2$ & $43 \pm 14$ & $18.3 \pm 0.4$ & $80.4 \pm 1.3$ & $11.2 \pm 2.7$ & $140 \pm 6$
\end{tabular}

Table 2. Polarisation measured in R Aqr over the regions showed in figure $1 \mathrm{~b}$

\begin{tabular}{ccccccc}
\hline Position & $\mathbf{P}(\%) \mathbf{H} \alpha$ & Angle & $\mathbf{P}(\%) \mathbf{G r}$ & Angle & $\mathbf{P}(\%) G g$ & Angle \\
\hline Center & $1.6 \pm 0.2$ & $124 \pm 3$ & $0.9 \pm 0.2$ & $134 \pm 2$ & $1.1 \pm 0.2$ & $145 \pm 4$ \\
Knot 1 & $4.9 \pm 2$ & $86 \pm 8$ & $17.4 \pm 5.5$ & $36 \pm 7$ & $8.1 \pm 0.9$ & $70 \pm 3$ \\
Knot 2 & $5.3 \pm 2.9$ & $110 \pm 11$ & $30 \pm 13$ & $172 \pm 9$ & $4.2 \pm 1.3$ & $15 \pm 6$ \\
Knot 3 & $\mathrm{~N} / \mathrm{A}$ & $\mathrm{N} / \mathrm{A}$ & $\mathrm{N} / \mathrm{O}$ & $\mathrm{N} / \mathrm{O}$ & $32 \pm 1$ & $70 \pm 1.2$ \\
Knot 4 & $4.5 \pm 2.9$ & $71 \pm 42$ & $\mathrm{~N} / \mathrm{O}$ & $\mathrm{N} / \mathrm{O}$ & $7.9 \pm 4$ & $160 \pm 10$ \\
Knot 5 & $39 \pm 21$ & $113 \pm 11$ & $\mathrm{~N} / \mathrm{O}$ & $\mathrm{N} / \mathrm{O}$ & $8.9 \pm 10$ & $160 \pm 26$
\end{tabular}
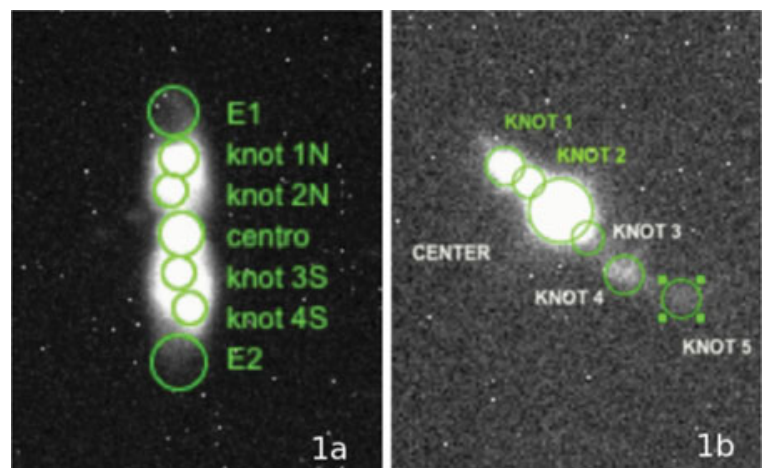

Figure 1. Regions where the polarisation were measured in M2-9 (1a), and in R Aqr (1b).

slightly on the radius of the region used to make the measurements. This is probably due to the low signal to noise in the R Aqr polarisation images.

\section{Conclusions}

We confirm the polarisation previously detected in M2-9 using the new polarimeter (POLIMA) at San Pedro Mrtir Observatory. In M2-9 we detected variation in the polarisation between the knots nearer to the central object and the farther ones on the jet. We report low polarisation detected on R Aqr. Only in one of the knots (the farther from the center) we detected substantial polarisation. This could imply dust scattered light.

These positive detections allow us to plan new polarimetric and spectro-polarimetric observations at higher resolution to find traces of magnetic field in these objects.

\section{References}

Castro-Carrizo, A., Neri, R., Bujabarral, V., et al. 2012, A\& A, 545, A1

Gledhill, T. M. 2005, MNRAS, 356, 883

Nichols, J. \& Slavin, J. D. 2009, ApJ, 699, 902

Scarrott, S. M. \& Scarrott, R. M. J. 1995, MNRAS, 277, 277

Solf, J. \& Ulrich, H. 1985, A\&GA, 148, 274 